\title{
Estrategias de implementación de sistemas de gestión del riesgo para las instituciones prestadoras de servicios de salud (IPS) en Colombia
}

\author{
Mara Claudia Arrieta Leottau \\ Universidad del Rosario \\ Colombia
}

Juan David Cabra Guzmán

Universidad del Rosario Colombia

Diana Marcela Martínez Sterling Universidad del Rosario Colombia 


\title{
Estrategias de implementación de sistemas de gestión del riesgo para las instituciones prestadoras de servicios de salud (IPS) en Colombia
}

\author{
Mara Claudia Arrieta Leottau ${ }^{D}$, Juan David Cabra Guzmán ${ }^{(D)}$, y Diana Marcela \\ Martínez Sterling \\ Universidad del Rosario - Colombia
}

Para citaciones: Arrieta Leottau, M., Cabra Guzmán, J., \& Martínez Sterling, D. (2021). Estrategias de implementación de sistemas de gestión del riesgo para las instituciones prestadoras de servicios de salud (IPS) en Colombia. Panorama Económico, 29(1), 24-47.

Recibido: 3 de octubre de 2020

Aprobado: 16 de diciembre de 2020

Autor de correspondencia:

Mara Claudia Arrieta Leottau

mcarrietale@gmail.com

Editor: Andrés Escobar E. Universidad de Cartagena-Colombia.

\section{Tipología IBN Publindex:}

Artículo de Revisión

Copyright: (C) 2021. Arrieta Leottau, M., Cabra Guzmán, J., \& Martínez Sterling, D. Este es un artículo de acceso abierto, distribuido bajo los términos de la licencia https://creativecommons.org/licenses/by-

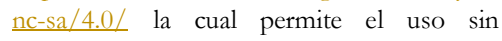
restricciones, distribución y reproducción en cualquier medio, siempre y cuando que el original, el autor y la fuente sean acreditados.

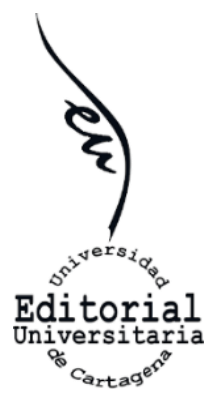

\section{RESUMEN}

La gestión del riesgo es un sistema organizado que permite prepararse para cualquier situación que eventualmente pueda afectar el funcionamiento organizacional. En Colombia, las Instituciones Prestadoras de Salud (IPS) en su mayoría se han limitado a la gestión de la seguridad del paciente, dejando a un lado, otros riesgos igualmente importantes, predisponiéndoles a detrimento patrimonial o reputacional. De otros sectores, es posible rescatar claves o estrategias para ser aplicadas en el sector salud. Este documento identifica estrategias de implementación de sistemas de gestión del riesgo para las instituciones prestadoras de servicios de salud en Colombia, a partir de una revisión exhaustiva y crítica de literatura con enfoque descriptivo. Se tuvieron en cuenta documentos a nivel nacional e internacional de carácter científico, técnico, y normativo relacionados con la gestión del riesgo en diferentes sectores. La implementación de un sistema de gestión de riesgo, otorga a las IPS herramientas para competir apropiadamente en el mercado de la salud, participar en cualquier RIAS, ya sea como prestador primario o complementario, adaptarse a los nuevos modelos de contratación y garantizar los ingresos operacionales. Por esta razón, se hace necesario que las IPS consideren la gestión de riesgos como el riesgo reputacional, el riesgo de gobierno corporativo, los riesgos de mercado, el riesgo del modelo de negocio, el riesgo de crédito, el operacional, los riesgos comerciales, el riesgo informático, el de corrupción y por supuesto el riesgo financiero. Por lo anterior, la implementación efectiva de la gestión del riesgo en salud, pero sin detrimento de la gestión de la enfermedad, es una necesidad manifiesta.

Palabras clave: Gestión clínica, gestión de riesgos, gestión en salud, riesgo a la salud, manejo de la enfermedad.

\section{Strategies for the implementation of risk management systems in institutions that provide health services (IPS) in Colombia}

\section{ABSTRACT}

Risk management is an organized system that allows the preparation for any situation that may eventually affect organizational performance. In Colombia, most of the Health Provider Institutions (IPS) have limited themselves to managing patient safety, leaving aside other equally important risks predisposing them to patrimonial or reputational detriment. It is possible to rescue keys or strategies to be applied in the health sector from other sectors. This document identifies strategies for the implementation of risk management systems in institutions that provide health services in Colombia, based on an exhaustive and critical review of the literature with a descriptive approach. Scientific, technical, and regulatory documents related to risk management in different sectors at a national and international level were reviewed. The implementation of a risk management system provides 
IPS with tools to compete appropriately in the health market, participate in any RIAS either as a primary or complementary provider, adapt to new contracting models and guarantee operational incomes. For this reason, it is necessary for IPSs to consider risk management such as a reputational risk, corporate governance risk, market risk, business model risk, credit risk, operational risk, commercial risk, IT risk, corruption risk and of course financial risk. Therefore, the effective implementation of health risk management, but without detriment to the management of the disease, is a manifest need.

Keywords: Clinical management, risk management, health management, health risk, disease management.

\section{INTRODUCCIÓN}

La gestión del riesgo es una secuencia organizada de actividades que pretenden modificar las consecuencias en el logro de un objetivo, por la posible materialización de una incertidumbre, procurando la identificación, el análisis, la evaluación, el tratamiento de esta, para anular, mitigar, o transferir ya sea la intensidad, la probabilidad o efectos de la amenaza identificada. Otros sectores como la banca, los seguradores, la ingeniería nuclear, el tecnológico- informático, el militar, la navegación, trabajan la gestión de riesgo hace muchos años, dada la tecnificación y modernización de los procesos que trajo la revolución industrial, permitiendo que pasaran de realizarse manualmente a automatizados, consiguiendo el mayor control de las actividades. Al acelerarse la elaboración de los distintos productos trajo un aumento en la siniestralidad, lo que llevó a la implementación de sistemas de gestión de calidad y a la aplicación de normas técnicas como la ISO 9001 e ISO 31000, interviniendo los posibles riesgos a nivel general de las diferentes organizaciones (ISOTools., 2018).

En Colombia es obligatorio para las entidades públicas, incluyendo las de salud, contar con políticas y sistemas para dar manejo apropiado a los riesgos, no así en las instituciones privadas (Presidencia de la República de Colombia, 2015). En las Instituciones Prestadoras de Servicios de Salud, sobre todo en aquellas de baja complejidad, no queriendo decir que las de mediana y alta complejidad se excluyan del problema, se ha enfocado la gestión del riesgo casi exclusivamente a lo relacionado con los riesgos en la prestación del servicio, favoreciendo la seguridad del paciente y prácticamente dejando a un lado la gestión de otros riesgos igualmente importantes, esto probablemente es debido a que la normatividad vigente sólo les obliga a implementar este tipo de acciones para disminuir el daño potencial a los usuarios, mientras que no es reglamentario para el resto de riesgos en los cuales pueden verse inmersos (Ministerio de Salud y Protección Social, 2019).

La gestión del riesgo no debe ser sólo un requisito más por cumplir, no es ocasional encontrar entre los distintos actores del SGSSS, documentos institucionales apropiadamente redactados y se siguen materializando los riesgos detectados previamente o peor aún, nunca antes contemplados, favoreciendo el detrimento patrimonial de las organizaciones por la aparición de demandas, daño de equipos, pérdida de información documental, deterioro de las estructuras física, fuga de cerebros, aumento del costo médico, esto teniendo en cuenta el aspecto netamente económico, si 
nos concentramos en los daños a las personas, no solo en su componente físico sino en el aspecto psicológico y moral, el abanico de posibles consecuencias negativas va desde prolongación de la estancia hospitalaria hasta la muerte pasando por secuelas que no solo dificultan su habitual desempeño dentro de la sociedad sino también el de su núcleo familiar y red de apoyo social, alterando definitivamente la economía del país por la disminución de horas laboradas.

Esta situación podría ser multicausal, en primer lugar por una identificación inadecuada de los riesgos, en segundo por inconsistencias en el análisis y la evaluación de los mismos y por tanto en la generación de estrategias apropiadas para su manejo y en tercer lugar, el cual se esperaría sea el menor de los casos, porque la implementación de estos sistemas de gestión del riesgo no han cumplido con la rigurosidad debida o peor aún se desconoce o no ha sido considerado importante, con este trabajo lo que se quiere es brindar a las instituciones prestadoras de servicios de salud, en especial a las de niveles más bajos de complejidad, recomendaciones claras y concisas de acuerdo a las experiencias de otros sectores que son muy exitosos en su gestión de riesgos, para que se pueda de esta manera evaluar su implementación por parte de las mismas.

El presente documento identifica las principales estrategias de implementación de sistemas de gestión del riesgo para las instituciones prestadoras de servicios de salud (IPS) en Colombia y se estructura de la siguiente manera: inicialmente se expone la normatividad vigente para la gestión del riesgo en salud en Colombia, posteriormente se define la gestión del riesgo en salud, su caracterización en otros sectores y se identificaron los modelos más ampliamente utilizados. Finalmente, se establecen los beneficios en las IPS de la implementación de estos sistemas, se analizaron las diferencias entre gestión del riesgo en salud y gestión de la enfermedad y se definen algunas claves para el diseño o rediseño del sistema de gestión de riesgo en las IPS de Colombia.

\section{NORMATIVIDAD VIGENTE EN LA GESTIÓN DEL RIESGO EN COLOMBIA PARA LAS INSTITUCIONES PRESTADORAS DE SERVICIOS DE SALUD}

Se proponen tres líneas para abordar la normatividad vigente, la primera relacionada con la gestión del riesgo, la segunda se refiere a la gestión del riesgo asociado a la prestación del servicio de salud, cuyo enfoque principal es la seguridad del paciente y la tercera, aborda la gestión del riesgo en salud en Colombia.

\section{Normativa general sobre gestión del riesgo}

Inicia en la Constitución colombiana refiriéndose a las competencias administrativas del estado, para hacer cumplir la ley y el fortalecimiento del bienestar común, con la garantía de las libertades, disfrute y ejercicio de deberes y derechos, así como de los servicios públicos entre otros, determinando que la actuación de aquellos en dicha función, debe estar dispuesta al bien general y fundamentadas en principios entre los cuales resaltan la eficacia y la economía (Constitución Política de Colombia, 1991, art 209); la Ley 87 de 1993, aplicable a todas las entidades y organismos estatales, incluyendo a las instituciones de salud públicas, determina la obligatoriedad de los sistemas de control interno enfocados a la protección de los recursos institucionales, instando a la apropiada administración de los diferentes riesgos que puedan afectarlos, por la implementación de medidas para 
prevenirlos o manejarlos una vez materializados (Congreso de la República de Colombia, 1993, p. art 2). En consecuencia, el Decreto 1083 de 2015, Decreto Único Reglamentario del Sector de Función Pública, dictamina que estos sistemas de control interno deben establecer políticas para el manejo o gestión del riesgo en unidad con la administración de las entidades públicas (Presidencia de la República de Colombia, 2015, art 2.2.21.5.4.), y determina la obligatoriedad de la implementación de las directrices emanadas por el Departamento Administrativo de la Función Pública (Presidencia de la República de Colombia, 2015, art 2.2.21.5.5.).

Estas directrices incluyen dos guías: la Guía Para la Administración del Riesgo, que establece la metodología e incluye una caja de herramientas para su aplicación, con la valoración de la institución frente a la gestión del riesgo: su plataforma estratégica y la gestión por procesos, la implementación de la política de administración del riesgo, la identificación del riesgo: evaluación de las condiciones que pueden afectar a la entidad, es decir, el contexto tanto interno como externo y la técnica para identificar el riesgo y por último, la valoración del riesgo que comprende: el análisis, la valoración y el monitoreo-revisión del mismo; transversal a estos pasos recomienda establecer mecanismos de comunicación a toda la entidad (Departamento Administrativo de la Función Pública, 2014).

La otra guía se denomina Guía Para la Administración del Riesgo y el Diseño de Controles en Entidades Públicas: Riesgos de Gestión, Corrupción y Seguridad Digital, la cual se constituye en una mejora a la anterior por unificar metodologías tanto para la administración del riesgo de gestión como de corrupción (Departamento Administrativo de la Función Pública, 2018).

Se podría inferir que la obligatoriedad de la implementación de sistemas de gestión o administración del riesgo es exclusiva para aquellas instituciones que pertenecen al sector público o público privado debido al manejo de recursos económicos de origen estatal.

Por ser Colombia un estado social de derecho (Constitución Política de Colombia, 1991) y que la salud en Colombia es considerada un servicio público (Congreso de la República de Colombia, 1993, art 4), es obligación del Estado garantizar el disfrute efectivo del mismo, razón por la que la Superintendencia Nacional de Salud adopta un modelo de Inspección, Vigilancia y Control enmarcado en la supervisión de los riesgos inherentes del sistema de salud, definiendo que todas las entidades vigiladas por ella deben establecer un sistema integrado de gestión de riesgo con unos lineamientos mínimos específicos emitidos por ella y exigibles en consecuencia a la emisión de los mismos (Superintendencia Nacional de Salud, 2018a).

Así las cosas, todos los vigilados están obligados a implementarlo, a la fecha, están definidos los lineamientos para las implementación en las Entidades Administradoras de Planes de Beneficio (EAPB) (Superintendencia Nacional de Salud, 2018b) y deben gestionar como mínimo los riesgos identificados como prioritarios por parte de la Supersalud que son: "Riesgo en Salud. Riesgo Actuarial. Riesgo de Crédito. Riesgo de Liquidez. Riesgo de Mercado de Capitales. Riesgo Operacional. Riesgo de Fallas del Mercado de Salud. Riesgo de Grupo. Riesgo Reputacional. Riesgo de Lavado de Activos y Financiación del Terrorismo" (Superintendencia Nacional de Salud, 2018b). 
También insta a la Administradora de los Recursos del Sistema General de Seguridad Social en Salud (ADRES) a implementar-actualizar su sistema teniendo como mínimos los siguientes riesgos: "I. Riesgo Operacional II. Riesgo de Lavado de Activos, Financiación del Terrorismo y Corrupción III. Riesgo de Crédito IV. Riesgo de Liquidez V. Riesgo de Mercado" (Superintendencia Nacional de Salud, 2018c); a la fecha de esta revisión, no están definidos los lineamientos para las IPS, cabe recordar que serán exigibles una vez emitidos. De manera esquemática puede revisarse esta primera línea de análisis en la figura.1

Figura 1. Gestión del riesgo en Colombia.

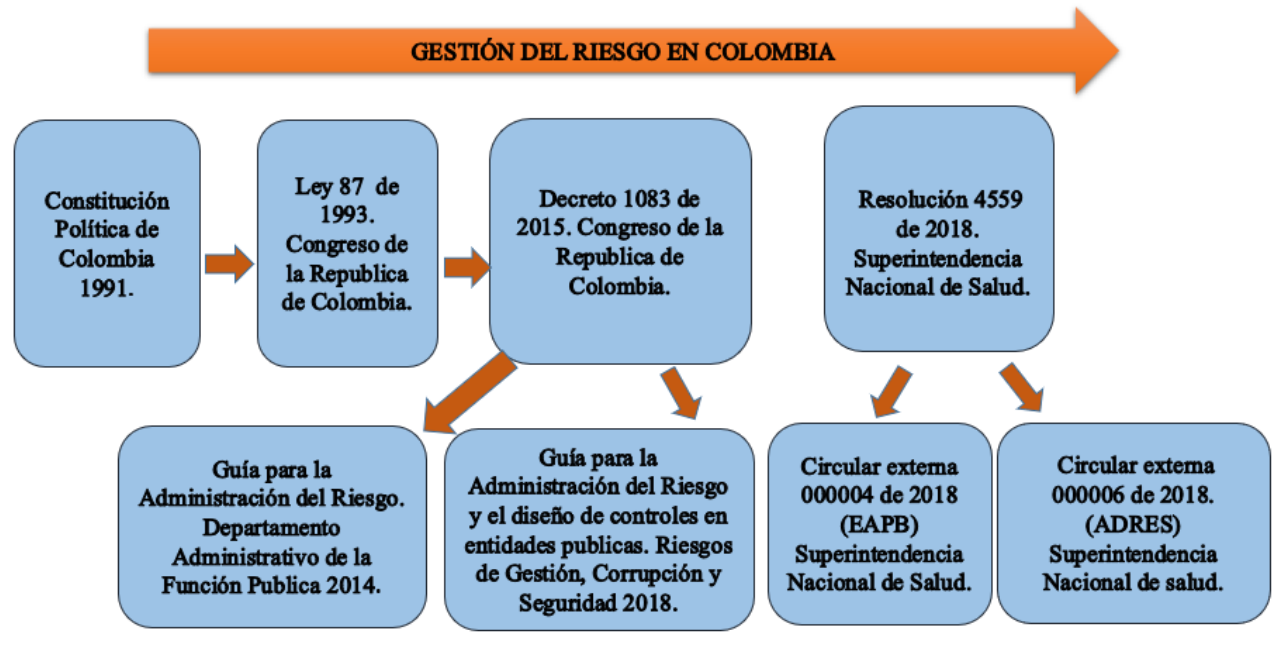

Fuente: Elaboración propia.

Normativa referente gestión del riesgo asociado a la prestación del servicio de salud

La contextualizamos a partir del Decreto Único Reglamentario del Sector Salud y Protección Social por el cual se encuentran obligadas a cumplir con el sistema único de habilitación tanto las EAPB como las IPS y que define "las condiciones básicas de capacidad tecnológica y científica, de suficiencia patrimonial y financiera y de capacidad técnico-administrativa, indispensables para la entrada y permanencia en el Sistema" (Presidencia de la República de Colombia, 2016, art 2.5.1.3.1.1.).

Para las EAPB su cumplimiento es evaluado por la Superintendencia Nacional de Salud (SNS) y busca minimizar la materialización de riesgos que puedan afectar la atención de los afiliados, el desempeño y sostenibilidad de la organización, obtener la autorización para operar el aseguramiento en salud en Colombia por cinco años, demostrando suficiencia en: 1. Capacidad técnico-administrativa: para poder asumir a cabalidad con las funciones de aseguramiento en aspectos de legalidad, administración, contabilidad, logística, suficiencia de recursos, incluyendo el talento humano. 2. Capacidad tecnológica: suficiencia estructural, tecnológica y en los sistemas de información para cumplir con las funciones del aseguramiento. 3. Capacidad científica: evidenciada por la apropiada representación del usuario, gestión del riesgo en salud y debida estructuración de la red integral de prestadores de servicios de salud (Ministerio de Salud y Protección Social, 2018, art 2.5.2.3.3.1.). 
Los lineamientos mínimos para demostrar la suficiencia según el manual de habilitación (Ministerio de Salud y Protección Social, 2018), son:

1. Sistema de gestión de riesgos.

2. Afiliación y libre elección en el SGSSS.

3. Atención del usuario e información para el afiliado.

4. Sistema de peticiones, quejas, reclamos, sugerencias, denuncias y tutelas.

5. Autorización de servicios médicos, medicamentos e insumos.

6. Fortalecimiento de la cultura de la seguridad social.

7. Gestión del talento humano.

8. Tecnologías de información.

9. Red integral de prestadores de servicios de salud.

10. Gestión de la salud pública.

11. Condiciones financieras de la entidad y gestión de los recursos del SGSSS.

12. Recaudo, compensación de aportes y liquidación de prestaciones contributivas.

13. Contratación y pago de servicios.

14. Gobierno organizacional.

Con respecto a la habilitación de las IPS, la resolución 3100 de 2019, define los servicios de salud que pueden ser ofertados, las condiciones a verificar y los estándares y criterios para la habilitación de estos servicios, el prestador debe cumplir con estos requisitos mínimos para garantizar la seguridad en el proceso de la atención para el usuario de servicios de salud.

El Sistema Único de Habilitación "busca controlar el riesgo asociado a la prestación de servicio de salud y a las condiciones en que éstos se ofrecen mediante el cumplimiento obligatorio de requisitos mínimos que dan seguridad a los usuarios" (Ministerio de Salud y Protección Social, 2019)

En el marco del Sistema Obligatorio de Garantía de la Calidad- SOGC, el componente de acreditación en salud hasta la fecha es el único opcional (Presidencia de la República de Colombia, 2016, art. 2.5.1.6.2). Con este Sistema Único de Acreditación en Salud se busca cumplir con los niveles de alta calidad en la atención bajo la dirección del estado, vigilado y controlado por la superintendencia de salud, incluye cuatro ejes principales: seguridad del paciente, humanización, gestión tecnológica y gestión del riesgo (Presidencia de la República de Colombia, 2016).

Tiene en total siete ejes trazadores que pretenden sobrepasar las expectativas y necesidades de los usuarios frente a la atención en salud, disminuir la posibilidad que el usuario sufra un evento adverso, fortalecer la atención humanizada, hacer una efectiva gestión del riesgo, fomentar la inclusión planeada de tecnología en las instituciones, favorecer una cultura organizacional orientada hacia la calidad, la transparencia y la ética y concretar acciones institucionales que contribuyan con la disminución de las desigualdades de las comunidades. (Ministerio de Salud y Protección Social, 2014, art. 2). La figura 2 nos presenta didácticamente esta información. 
Figura 2. Gestión del riesgo asociado a la prestación del servicio de salud en Colombia

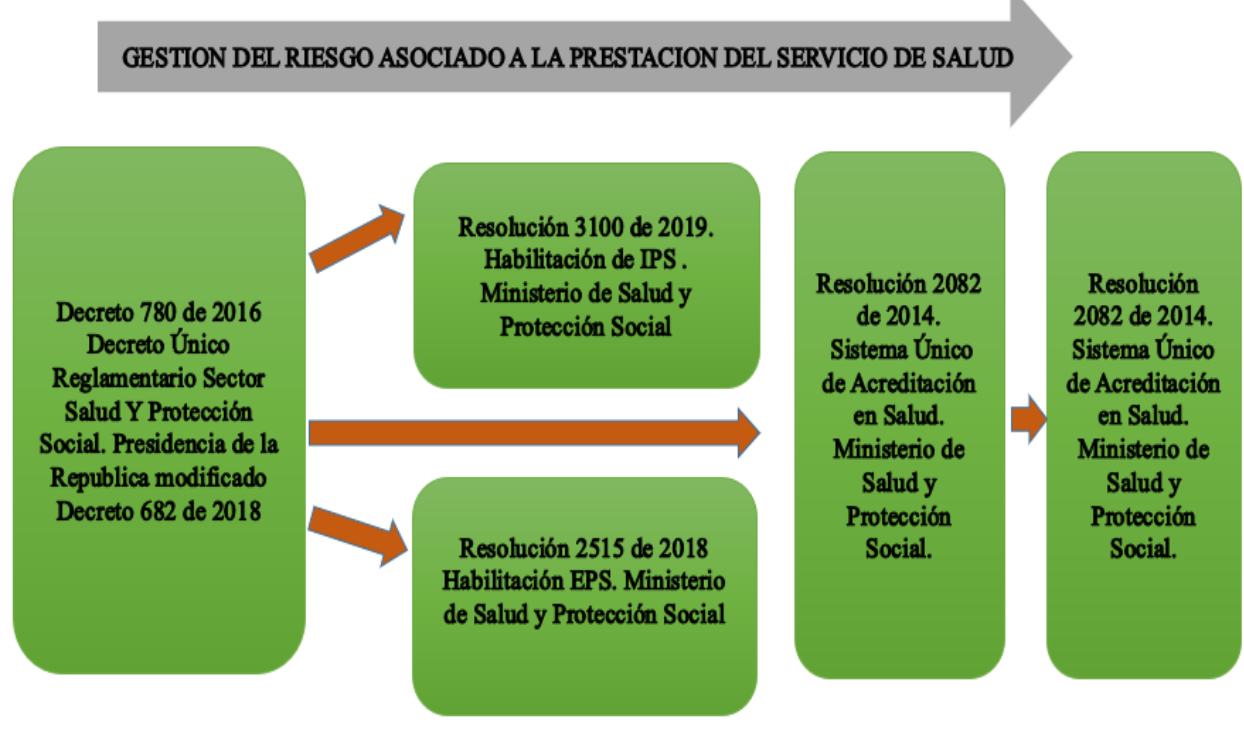

Fuente: Elaboración propia

\section{Normatividad gestión del riesgo en salud en Colombia}

El sistema de seguridad social en salud, creado por la Ley 100 de 1993 y modificado por las Leyes 1122 de 2007 y 1438 de 2011 busca proteger a las personas y comunidades de las situaciones que puedan afectar el disfrute de los derechos irrenunciables, procurándoles una calidad de vida con la dignidad de ser humano (Congreso de la República de Colombia, 1993), estableciendo como principal estrategia al aseguramiento, que asume para la población afiliada su representación, el acceso a servicios de salud articulados y con calidad, la gestión del riesgo financiero y del riesgo en salud (Congreso de la República de Colombia, 2007), procurando un modelo de atención enmarcado en la atención primaria en salud coordinada con los actores del sistema, en pro del mejoramiento de la salud y ordena un Plan Decenal de Salud Pública-PDSP (Congreso de la República de Colombia, 2011).

El Plan Decenal de Salud Pública 2012-2021 determina ocho dimensiones prioritarias y dos dimensiones transversales. Las prioritarias son: 1) salud ambiental, 2) vida saludable y condiciones no transmisibles, 3) convivencia social y salud mental, 4) seguridad alimentaria y nutricional, 5) sexualidad, derechos sexuales y reproductivos, 6) vida saludable y enfermedades no transmisibles, 7) salud pública en emergencias y desastres y 8) salud y ámbito laboral. Las transversales son: gestión diferencial de las poblaciones vulnerables y el fortalecimiento de la autoridad sanitaria para la gestión de la salud; cada una de estas dimensiones tienen definidos unos resultados en salud y a su vez unas acciones que deben ser ejecutadas por medio de tres líneas operacionales: la promoción de la salud, la gestión de riesgo en salud y la gestión de la salud pública. (Ministerio de Salud y Protección Social, 2013b).

Siendo responsabilidad del Estado, según la Ley Estatutaria en Salud, garantizar en lo individual y lo colectivo el derecho fundamental a la salud, es decir, el suministro de los servicios de salud en los niveles y complejidad requeridos, con la disponibilidad, 
accesibilidad, oportunidad, eficacia y continuidad apropiada para cada situación, desde la promoción y prevención hasta la rehabilitación y paliación, así como la disminución de la desigualdad por la intervención de los determinantes sociales que afectan la salud, para poder hacerlo tiene que procurar un sistema de salud articulado con las directrices y políticas, tanto de manera individual como colectiva con sostenibilidad financiera y con un oportuno y suficiente flujo de recursos económicos (Congreso de la República de Colombia, 2015).

El Plan Nacional de Desarrollo (PND) 2018-2022 Pacto por Colombia. Pacto por la Equidad, se encuentra conformado por pactos estructurales: 1. Pacto por la legalidad 2. Pacto por el emprendimiento, la formalización y la productividad 3. Pacto por la equidad que incluye la mejora de la salud de la población y su satisfacción en consonancia con la sostenibilidad y la equidad (Congreso de la República de Colombia, 2019).

En este sentido se presenta la modificación de la Política de Atención Integral en SaludPAIS del país y se cambia al nuevo Modelo de Atención Integral Territorial-MAITE, ratificando la política que centra toda la atención en el ciudadano, para garantizar el disfrute efectivo del derecho fundamental a la salud; buscando conseguir las mejores condiciones de bienestar para la población, articulando a todos los integrantes del sistema y la sociedad. La PAIS se desarrolla con las siguientes estrategias y enfoques 1) Estrategia de atención primaria en salud. 2) Estrategia de gestión integral de riesgo en salud. 3) Enfoque de cuidado a la salud 4) Enfoque diferencial de derecho. Siendo la estrategia de gestión integral de riesgo en salud transversal a todos, pues pretende que el sistema se pueda anticipar a las patologías y a los traumatismos que puedan materializarse en los usuarios o que al menos puedan ser detectados y abordados oportunamente para minimizar las secuelas, disminuir los periodos de rehabilitación y las consecuencias de la enfermedad en la calidad de vida de las personas.(Ministerio De Salud y Protección Social, 2019)

El MAITE operativiza la política y busca garantizar a los ciudadanos el goce del derecho a la salud, brindando a la autoridad sanitaria territorial las fortalezas necesarias, la articulación de los diferentes actores del sistema, así como aumentar los niveles de salud de la población, la satisfacción de los usuarios y la sostenibilidad financiera. El modelo tiene ocho líneas de acción que son: 1) aseguramiento, 2) salud pública, 3) prestación de servicios de salud, 4) talento humano en salud, 5) financiamiento, 6) enfoque diferencial, 7) intersectorialidad, 8) gobernanza; cada una de ellas con unas acciones específicas y unas herramientas dentro de un marco normativo que le permitirán ejecutar las acciones. La formulación del Plan de Acción Implementación del MAITE (PAIM), el cual sería la materialización del MAITE en los entes territoriales con la incorporación de las acciones implementadas en el en el modelo anterior MIAS, estaba prevista iniciar con la posesión en 2020 de los alcaldes y gobernadores y tendría 6 meses para su planeación, formulación y adopción, una vigencia de ejecución de cuatro años y el monitoreo lo haría el Ministerio de Salud y Protección Social-MSPS por informes trimestrales de cada ente territorial acerca del cumplimiento de los compromisos establecidos en la PAIS; el PAIM podría ajustarse en el penúltimo trimestre de cada año por los entes territoriales con la oportuna comunicación al MSPS (Ministerio De Salud y Protección Social, 2019). Debido a la pandemia por SARS-CoV-2 se amplían los plazos, por lo que esta cartera generará una metodología para la formulación del PAIM y los entes gozarán de un plazo 
de un año de plazo para la formulación del mismo a partir de la emisión del lineamiento (Ministerio de Salud y Protección Social, 2020).

Para favorecer el cumplimiento de la política y del modelo, los entes territoriales están encargados de realizar el Plan Territorial de Salud (PTS), por la aplicación de una metodología analítica- sintética, que permite la caracterización poblacional, es decir, identificar los riesgos y determinantes de la salud que afectan a la población, surgiendo el Análisis de Situación de Salud - ASIS; el PTS debe estar enmarcado en las siguientes líneas: promoción de la salud, gestión de riesgo en salud y gestión de la salud pública (Ministerio de Salud y Protección Social, 2015a); igualmente el Plan de Salud Pública de Intervenciones Colectivas (PIC) estará definido por el contexto particular de cada ente territorial, la priorización en salud realizada de acuerdo con el Plan Decenal de Salud Pública vigente, las estrategias establecidas en su PTS y lo pertinente de acuerdo con las competencias establecidas en la ley para cada entidad. (Ministerio de Salud y Protección Social, 2015a); y queda definida que para el ente territorial la gestión del riesgo en salud es el proceso que le permite a la entidad territorial identificar, analizar e intervenir riesgos colectivos en salud, así como la percepción de la población frente a las amenazas y vulnerabilidades, y afrontar la incertidumbre, que consiste en disminuir o mantener la ocurrencia de eventos negativos para la salud en niveles socialmente aceptables a través de estrategias de prevención o mitigación (Ministerio de Salud y Protección Social, 2015a).

Para ejecutar y materializar la PAIS y el MAITE, las EAPB en Colombia, deben realizar anualmente la caracterización poblacional de sus afiliados, utilizando la metodología para levantar el análisis de situación de salud- ASIS, esto para detectar riesgos y priorizar estos grupos poblacionales dentro de la población afiliada (Ministerio de Salud y Protección Social, 2015b). Así mismo, las aseguradoras deben suministrar la información complementaria, definida por el MSPS, en el Sistema de Información Transaccional (SAT) para la identificación y gestión de los riesgos de los afiliados. (Presidencia de la República de Colombia, 2016, p. art. 2.1.3.3.), de la misma forma deben tener un sistema de gestión del riesgo en concordancia con la Ley Estatutaria en Salud, el PND, el PDSP, la PAIS y demás normas relacionadas. (Presidencia de la República de Colombia, 2016, p. art. 2.5.2.3.3.2.)

Este sistema de gestión integral del riesgo, para las EAPB debe estar centrado en la gestión del riesgo en salud y permitirles identificar, evaluar, medir, seguir y monitorear los riesgos, favoreciendo el mejoramiento continuo y la innovación, aumentando la satisfacción de los usuarios y los indicadores de resultados en salud. Para esto, deben diseñar e implementar un modelo de atención en salud que identifique y clasifique el riesgo en salud, manteniendo esta información actualizada, con esta información diseñar e implementar Rutas Integrales de Atención en Salud- RIAS para los grupos priorizados, las cuales serán ejecutadas por las Redes Integrales de Prestadores de Servicios de SaludRIPSS, previamente organizadas y autorizadas, los equipos de salud interdisciplinarios y capacitados en el modelo y la estrategia, además, deben organizar la demanda de la prestación del servicio anticipándose a las necesidades inclusive no percibidas del usuario y fortaleciendo la referencia y contrareferencia, también deben fomentar contratos con los prestadores que favorezcan la orientación a los resultados en salud, el diseño de instrumentos que faciliten la disponibilidad de la información de los afiliados, la utilización de la nota técnica como herramienta de gestión del riesgo, evaluación y análisis de los 
indicadores de las RIAS y la mejora continua y decisiones basadas en el desempeño de procesos. (Ministerio de Salud y Protección Social, 2016b).

La modificación de la PAIS y la implementación de la MAITE permanece apoyada en la materialización de las RIAS que son la búsqueda de alinear las condiciones para que sea integral, para cada usuario, su familia y las poblaciones, la atención en salud por parte de cada uno de los integrantes del SGSSS mejorando por tanto los resultados en salud y disminuyendo el estado de enfermedad de la población, esto debido a que cada una de ella integra y organiza la secuencia de las distintas acciones. Las RIAS pueden ser: para la promoción y mantenimiento de la salud, de grupos de riesgo y de eventos específicos y contienen múltiples acciones a desarrollarse en diferentes entornos: hogar, laboral, comunitario o educativo, las intervenciones pueden ser individuales, colectivas o poblacionales y la finalidad puede variar entre la gestión de la salud pública, la promoción de la salud, el diagnóstico, el tratamiento, la rehabilitación o la paliación y la decisión de la implementación de estas intervenciones puede estar basada en la evidencia científica, en la experiencia o en la evidencia de las comunidades objetivo. Las EAPB deben implementarlas articuladamente previa adopción y adaptación de las mismas, de acuerdo con el territorio y su población afiliada, integrando a las IPS en este proceso, para poder garantizar que los usuarios se beneficien efectivamente de los servicios de salud tanto primarios como complementarios, todo esto bajo la asesoría, acompañamiento y vigilancia de los entes territoriales quienes deben reportar a la Superintendencia nacional de Salud en caso de incumplimiento por parte de estas. (Ministerio de Salud y Protección Social, 2016b, p. art. 5)

La participación de las IPS en la gestión del riesgo en salud se evidencia en su participación en las RIPSS, organizadas y habilitadas por las EAPB, por el establecimiento de contratos con las EAPB y la materialización de los mismos para beneficio de los usuarios (Ministerio de Salud y Protección Social, 2016b) y a través del sistema obligatorio de garantía de la calidad básicamente, que hemos explicado previamente, dado que ellas se constituyen en las ejecutoras de las acciones planeadas en las RIAS (figura 3).

Figura 3. Gestión del riesgo en salud en Colombia

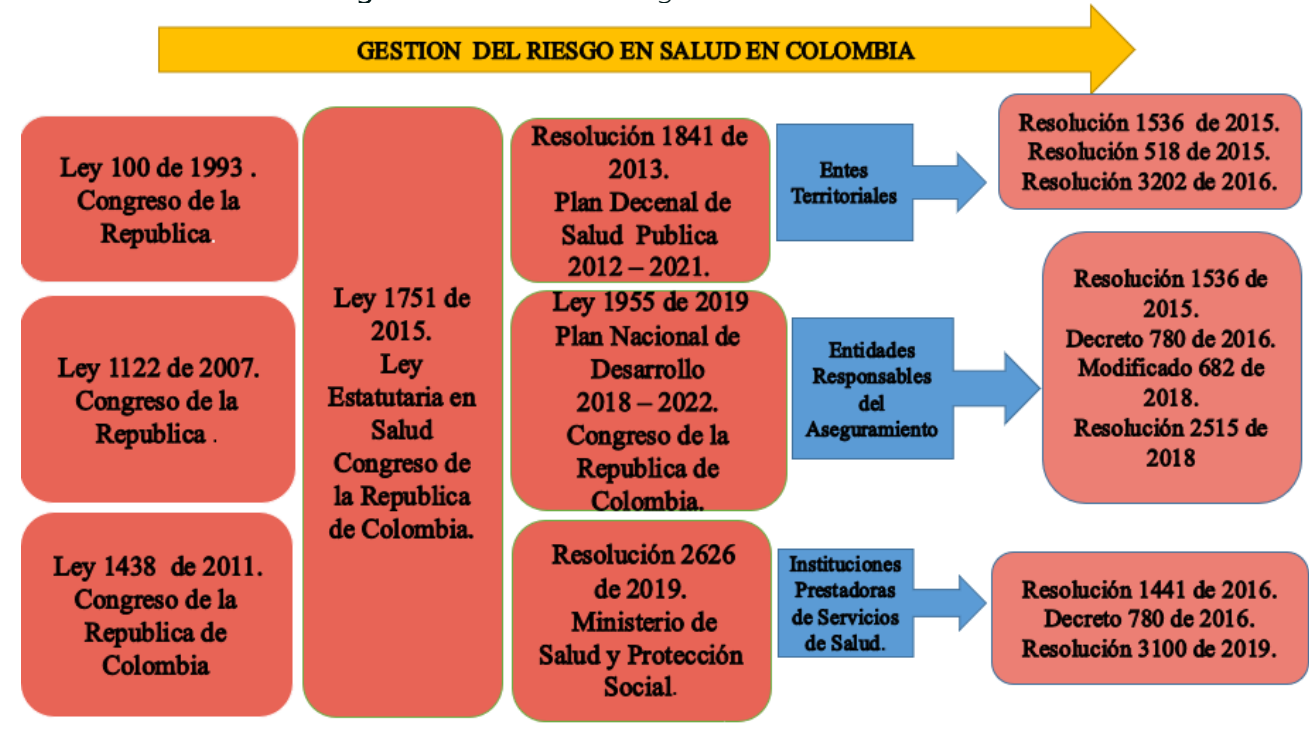

Fuente: Elaboración propia. 


\section{GESTION DEL RIESGO: DEFINICIONES}

El concepto de riesgo se liga a la idea de exposición a cambios, donde se mantiene la incertidumbre, que puede conllevar resultados negativos o positivos a corto, mediano o largo plazo en el logro de los objetivos, sabiendo que, a mayor riesgo, mayor será el rendimiento de las inversiones (Nunes, 2009, p. 4); para que un riesgo sea controlable se deben considerar tres características: incertidumbre, impacto y tiempo (Smith et al., 2002). La incertidumbre es una probabilidad desconocida, el impacto que puede ser una pérdida o ganancia financiera y el tiempo la duración de la exposición al riesgo. El riesgo es inherente a cualquier decisión, los cambios se presentan constantemente, las tecnologías avanzan y las ganancias fluctúan a lo largo del tiempo (Crouhy et al., 2008). La gestión del riesgo por tanto serán todas las acciones organizacionales orientadas a anticipar eventos de pérdida o ganancia, utilizando herramientas que permitan identificar, gestionar y protegerse contra dichos riesgos. (De Oliveira et al., 2008, p. 175).

Identificar los diferentes tipos de riesgo a los que está expuesta una organización es vital y de acuerdo con el sector en que se desarrolle pueden identificarse o clasificarse de diferente manera. La Federación de la Asociación Europea de Gestión de RiesgosFERMA, considera que los riesgos pueden ocasionarse por factores internos o externos a la empresa, y los clasifica como riesgos financieros, estratégicos, operacionales y de azar (Giraldo \& Núñez, 2020). Se presentan diferentes tipos de riesgos comerciales: riesgos estratégicos que afectan los objetivos que pretenden ganar competitividad, relacionados con la planificación fallida o ineficaz (Marshall, 2002, p. 20); riesgos de gobierno corporativo que atañen a los accionistas y directivos, su interrelación que puedan desencadenar comportamientos antiéticos, abusos de poder o fraudes (Instituto Brasileiro De Governança Corporativa, 2007, p. 19); de la estrategia de mercado o riesgo del modelo de negocio que se relaciona a la pérdida de ingresos debido a diversas situaciones como la toma de una decisión errónea, la incertidumbre sobre la demanda de un producto, el costo de producción y su acogida en el mercado, lo cual depende del éxito o fracaso de un producto y el gusto de los clientes (Rodrigues Pereira, 2014).

Igualmente son considerados aquellos como el riesgo de imagen que corresponde a situaciones que puedan afectar la reputación de una empresa, como una propaganda negativa, una noticia falsa y traducirse en pérdidas financieras y o generar disminución en la credibilidad de la calidad del producto o servicio (Assi, 2012, p. 42); los riesgos financieros que corresponden a una mala gestión y administración no controlada y pueden presentarse resultados desfavorables para la organización (Rodrigues Pereira, 2014); el riesgo de mercado como aquella exposición a una variabilidad desfavorable en costos o retornos dados por cambios en tasas de interés, tasas de cambio y la fluctuación de los precios de acciones y productos que llevan a perdidas en el valor de activos y pasivos (Assi, 2012, p. 44).

Adicionalmente, se considera el riesgo de crédito como incumplimiento del pago de las obligaciones económicas adquiridas (Gil et al., 2013, p. 134); el riesgo de liquidez que en opinión de Jorion (1998, p. 15), se divide en riesgo de liquidez de mercado/producto (incapacidad de ejecutar una transacción a precio de mercado) y riesgo de liquidez de flujo de efectivo /recursos (gestión ineficaz de flujo de caja de la empresa o un pronóstico de costos deficientes, ocasionado por la incapacidad de cumplir con los pagos y una falta 
de financiación). También se encuentran los riesgos operacionales, como fallas de gestión, fraude y error humano que generan pérdidas potenciales (Crouhy et al., 2008, p. 26); el riesgo de proceso donde la empresa no cuenta con una secuencia organizada de sus funciones y actividades llevando al desperdicio de recursos propios (Hammer et al, 1994, p. 24); el riesgo de personal como las pérdidas financieras o en el logro de los objetivos se deben a fallas humanas no intencionales (Assi, 2012, p. 42); el riesgo de eventos externos que se corren en virtud de desastres naturales que pueden interrumpir la labor o destruir las instalaciones físicas (Assi, 2012, p. 42); riesgo de cumplimiento que según Assi (2012, p. 43) es el incumplimiento o violación de regulación y normas legales. Por otro lado, también se contempla el riesgo legal o reglamentario como las pérdidas económicas o reputacionales a partir de potenciales de sanciones judiciales que pueden afectar tanto a clientes, proveedores, accionistas (Zeno, 2007, p. 28); el riesgo contable cuando trabajadores de la empresa incumplen con principios que pueden generar sanciones, demandas y una reputación contaminada por acciones fraudulentas, errores de comunicación y discrepancias (Assi, 2012, p. 45).

\section{GESTION DEL RIESGO EN EL SECTOR ASEGURADOR}

En el sector asegurador, los métodos más comunes para la evaluación de riesgos se basan según Barros y de Madrid (2011) en modelos estáticos o dinámicos. En los estáticos implica que el análisis de la posición financiera se realiza en un momento, considerando para evaluar la solvencia: las cuentas del balance, las partidas integrantes de la cuenta de pérdidas y ganancias o la exposición a riesgos subyacentes de las inversiones. Los dinámicos toman en cuenta un periodo y se basan en proyecciones de flujos de caja y pueden clasificarse en modelos basados en escenarios (evalúan la sensibilidad de la entidad al impacto de diversos escenarios en la valoración de sus activos) o en principios (no existen pautas sobre el sistema a emplear para cuantificar sus necesidades de capital, cuya valoración se basa en la utilización de modelos internos que siguen ciertos principios prefijados). Por su parte, los modelos de base probabilista o stress testing, evalúan un número definido de probabilidades estadísticas para determinar cuál es la peor combinación de parámetros y la pérdida que esa combinación produciría, intentando cubrir toda la gama de variables de riesgo reproducidas a través de distribuciones estadísticas y procedimientos de simulación (Barros \& de Madrid, 2011).

También son utilizados modelos deterministas (las predicciones se basan en como los procesos actúan para una situación dada, y fueron utilizados inicialmente para eventos físicos o biológicos) y probabilísticos (basados en la probabilidad de predecir como los parámetros financieros clave interactúan entre ellos en el tiempo y generan una distribución de resultados basados en simulaciones de dichos parámetros en el futuro). Los modelos internos proponen una definición y una medición de la necesidad de capital en función de las características de la actividad de la empresa. Sin embargo, estos provocan una serie de dificultades porque no ofrece una cifra exacta, sino que estudia acontecimientos extremos cuya probabilidad de que sucedan es escasa, y puede no ser suficiente para su evaluación. (Barros \& de Madrid, 2011).

Nace un modelo de riesgos fundamentales con un principio de inversión buscando una cartera que haga que sea rentable para un riesgo explícito o llevar a un mínimo riesgo el modelo para su gestión en la aseguradora, debe cubrir el ciclo de negocio; lo primero es 
tener un orden de cobertura, gestionar el siniestro, siempre hay un riesgo, donde de primera mano se realiza una inversión (Becerra \& Semper, 2007).

\section{GESTION DEL RIESGO EN EL SECTOR BANCARIO}

Otro sector que históricamente ha gestionado su riesgo es el bancario y financiero, en 1974 se conforma el Comité de Supervisión Bancaria de Basilea, integrado por los gobernadores de los bancos centrales del G10 encargado de generar estándares de supervisión para la banca (Gaviria \& Lemarie, 2008). En 1988, de acuerdo con Jiménez Rodríguez y Martín Marín (2005) se publica el acuerdo Basilea I, concertando un capital mínimo para cualquier entidad financiera, en concordancia con los riesgos que enfrentan. Según el comité "El riesgo operacional se define como el riesgo de pérdida debido a la inadecuación o a fallos de los procesos, el personal y los sistemas internos o bien a causa de acontecimientos externos" (Arbeláez et al., 2006). Se clasifica dicho riesgo en tres categorías: riesgo de mercado, crediticio y operacional. En 2004 se publica un nuevo acuerdo: Basilea II, que es una revisión del capital inicial requerido para asegurar las instituciones bancarias ante los riesgos operativos y financieros (Arbeláez et al., 2006); este capital debe ser suficiente para los riesgos de crédito, mercado y tipo de cambio (Pérez-Fructuoso, 2014), se orienta la normativa a regular en tres aspectos claves: los requisitos financieros mínimos, el análisis cualitativo del riesgo y medidas para reforzar la disciplina del mercado, valorando niveles de solvencia, capitales necesarios, acciones apropiadas para gestionar el riesgo (Pérez-Fructuoso, 2014).

En Colombia en 2006, la Superintendencia Financiera introduce la Circular Externa 048, dando instrucciones sobre la adopción de un Sistema de Administración de Riesgo Operativo (SARO) para iniciar o adaptar los sistemas de administración de riesgo operacional para los establecimientos bancarios colombianos (Jiménez Rodríguez \& Martín Marín, 2005).

\section{METODOLOGÍAS UTILIZADAS PARA LA GESTIÓN DEL RIESGO}

Según Vega de la Cruz et al. (2017), se presentan ocho diferentes metodologías para la gestión del riesgo que se describen a continuación: metodología de análisis y de riesgos de los sistemas de información: se relaciona con todo el sistema informático; la metodología giro: es aplicable a cualquier amenaza no cuenta con destinatario absoluto se busca tener una calidad total. El método Octave: es la comprensión de los recursos que afectan la seguridad de una organización es genérico e independiente basado en las necesidades que tenga la misma. Así mismo, Risie universe: identifica los riesgos más importantes para la empresa o para lograr los objetivos estratégicos integrados como son la evaluación de la gestión del talento humano.

Del mimos modo describe el modelo pulmón: busca generar y preservar el valor en la empresa manteniendo la organización a largo plazo logrando el bienestar general y el mejoramiento de la calidad, el análisis funcional de operatividad que se diseñó por seguridad de la información para analizar y garantizar el riesgo desde los procesos, el Trademey: enmarca e incluye el control interno y el chinchorro de gestión: basado en el arte de redes, que logra incluir técnicas estadísticas que tratan los riesgos organizacionales. Las redes que revisa son las Redes de Petri: para sintonizar y aportar 
bases para analizar formas de un sistema modulado, las redes de influencia: para calificar las variables, las redes sociales: grupos de personas que buscan un objetivo común y se entrelaza con la dirección del vínculo, redes de transición: simbolizan las probabilidades de cambio y redes bayesianas: accionan las interdependencias para que las empresas actúen contando con un grupo de expertos metodológicos. El Informe del Comité de Organizaciones Patrocinadoras de Treadway-COSO, permite obtener información para formular la evaluación del control interno de la entidad, al identificar los procesos con mayores niveles de riesgos, las acciones y actividades se dirigirán hacia estos. (Sánchez, 2016). Es necesario la participación de los colaboradores para el éxito de la gestión del riesgo, ya que la comprensión de los factores de decisión que involucra dará su mayor efectividad sí es conducido por personas que están cerca de las situaciones de riesgo en el negocio y comprenden con facilidad los factores que los rodean y sus consecuencias. (Sánchez, 2016)

La Norma Técnica Colombiana NTC- ISO 31000:2011 se enfoca en la gestión del riesgo, delimitando claramente los principios y directrices. Analiza el hecho que cada organización se encuentra sometida a la influencia de factores tanto internos como externos que pueden modificar el logro de los objetivos propuestos por lo que define el riesgo como "el efecto de la incertidumbre sobre los objetivos"(Instituto Colombiano de Normas Técnicas y Certificación. ICONTEC, 2011, p. 4). Plantea la importancia para la organización de la adecuada determinación del contexto tanto interno como externo: objetivos, entorno, actores, criterios de riesgo, que le permitan identificar los diversos riesgos a los cuales se expone y poder evaluar el origen y la complejidad de estos; puede ser utilizado por cualquier empresa por no ser específica para ningún sector. El proceso para la gestión del riesgo cuenta con cinco actividades, la primera y la última transversales a la otras: comunicación y consulta, establecimiento del contexto, valoración del riesgo que incluye en orden la identificación del riesgo, el análisis y la evaluación del riesgo, tratamiento del riesgo y el monitoreo y revisión con el debido registro de cada una de las actividades realizadas. (Instituto Colombiano de Normas Técnicas y Certificación. ICONTEC, 2011).

\section{BENEFICIOS DE LA IMPLEMENTACIÓN DE UN SISTEMA DE GESTIÓN DEL RIESGO EN LAS INSTITUCIONES PRESTADORAS DE SERVICIOS DE SALUD EN COLOMBIA}

Un sistema integral de gestión que incluya manejo de riesgos estratégicos, riesgos de imagen o reputacionales, riesgos operativos, financieros, riesgos de cumplimiento, los asociados a la tecnología e inclusive los riesgos de corrupción generarán múltiples beneficios para la organización. (Departamento Administrativo de la Función Pública, 2014).

El Instituto Colombiano de Normas Técnicas y Certificación- ICONTEC, en la NTC ISO 31000:2011 (2011), señala que le permite a la organización los siguientes beneficios:

1. Una mayor posibilidad de conseguir los objetivos planteados: al tener apropiadamente identificados los riesgos y las medidas para controlarlos efectivamente implementadas en diferentes procesos.

2. El desarrollo de la organización desde una actitud propositiva, anticipándose a la materialización de amenazas que puedan deteriorar los resultados institucionales. 
3. Mantenerse en el pleno cumplimiento de requisitos legales exigidos para su ejercicio.

4. Incrementar su desempeño organizacional, tanto en eficacia como en eficiencia, por el mejoramiento en los controles, la asignación apropiada de recursos para la gestión del riesgo, la presentación de información y el aumento de la confianza en esta para la toma de decisiones y la planeación institucional.

5. Maximizar ganancias económicas y disminución de pérdidas por costos de no oportunidad, accidentes- incidentes laborales, eventos adversos o deterioro ambiental.

Específicamente en Colombia, para las IPS, su implementación es apalancada para poder competir apropiadamente en el mercado de la salud, participar en cualquier RIAS, ya sea como prestador primario o complementario; adaptarse al modelo de contratación, garantizando los ingresos operacionales de cada IPS han ido migrando, actualmente se encuentra casi extinto el modelo de pago por servicio o pago por evento, reservado para aquellas situaciones excepcionales y poco frecuentes, siendo reemplazado por modelos de contratación que favorecen el pago anticipado de montos globales por la atención en salud, que son sustancialmente menores y que buscan favorecer la efectiva gestión del riesgo técnico por parte de las IPS y cuya conveniencia radica en el volumen de pacientes a atender y la oportunidad en el pago anticipado de los mismos; en los modelos de contratación están los pagos por capitación, pagos por conjunto integral de atenciones, por paquete, por grupos relacionados (Ministerio de Salud y Protección Social, 2017b) o pagos globales prospectivos, estos modelos de contratación son la opción más viable y están enmarcados en la política y modelo de atención de salud vigente en Colombia (Ministerio De Salud y Protección Social, 2019), por lo que si una IPS no está haciendo apropiadamente la gestión de su riesgo, será al corto - mediano plazo insostenible financieramente, por el desgaste administrativo y desperdicio de recursos en la atención de la materialización de los riesgos, que no le permitirá enfrentar estos modelos de contratación.

Un riesgo que ha sido descuidado históricamente por las instituciones prestadoras de servicios de salud, es el riesgo reputacional, aquellas instituciones cuyo nombre representa o garantiza tranquilidad, cumplimiento, seguridad hoy en día en Colombia están siendo prósperas económicamente, mientras que aquellas que por diversos motivos han caído en desprestigio, con razón o sin ella, a pesar de todos los esfuerzos que hacen por lograr los objetivos institucionales realmente se encuentran con una barrera frente el usuario final del servicio de salud y de sus representantes que son las EAPB, como una de sus principales funciones de aseguramiento, mejor fama, mejores contratos, esto de especial cuidado en nuestra época de avances digitales y comunicación instantánea.

\section{GESTIÓN DEL RIESGO EN SALUD: DEFINICIÓN Y DIFERENCIAS CON GESTIÓN DE LA ENFERMEDAD, DESDE LA PERSPECTIVA DE LAS INSTITUCIONES PRESTADORAS DE SERVICIOS DE SALUD.}

La mayor carga de enfermedades crónicas, la inversión progresiva de la pirámide poblacional que experimenta el país, la llegada de tecnologías innovadoras para la atención de las mismas a mayores costos, entre otros, exigen que se prioricen estrategias 
que mejoren la calidad de vida de los usuarios, su experiencia en el proceso de atención y mejores resultados en salud para la población, es importante tener claros los conceptos y las diferencias de gestión de la enfermedad y gestión del riesgo en salud.

\section{Gestión de la enfermedad}

En la década de los noventa desde la industria farmacéutica de los Estados Unidos, como respuesta a medidas para contención de costos impuestas por las Health Maintenance Organizations - HMO (organizaciones para el mantenimiento de la salud) y por el gobierno en turno para el control de los precios de sus productos y siendo que las estrategias comerciales para incentivar la venta de los medicamentos y dispositivos médicos tradicionalmente utilizadas como la visita médica y las negociaciones con las HMO y pharmaceutical-benefits management- PBM (empresas de beneficios farmacéuticos) no conseguían los resultados esperados, surgen los programas para la gestión de enfermedades (Bodenheimer, 1999).

Un programa de gestión de enfermedades de acuerdo con Care Continuum Alliance CCA (Alianza para el cuidado continuo), que en sus inicios se denominó Disease Management Association of América -DMAA (Asociación Americana de gestión de la enfermedad) es "un sistema de intervenciones y comunicaciones coordinadas de atención médica para poblaciones con afecciones donde los esfuerzos de autocuidado de los pacientes son significativos" (Care Continuum Alliance (CCA), s. f.), en otras palabras, el manejo de la enfermedad es la búsqueda de la mejora en los resultados tanto en términos de salud, bienestar y económicos por el incremento de la calidad de atención en programas coordinados con distintos actores del sistema de salud para la atención durante el curso de la enfermedad, generalmente crónica, con intervenciones basadas en la mejor evidencia clínica disponible y estrategias de educación al paciente, la familia y al personal de la salud que favorezcan el autocuidado y la consulta oportuna por la identificación de signos y síntomas de complicaciones o exacerbaciones (Care Continuum Alliance (CCA), s. f.).

Estos programas de gestión de la enfermedad han evolucionado desde su implementación en básicamente dos vertientes: carve-out o modelo fragmentado que consiste en la contratación de organizaciones para la atención de patologías crónicas y por tanto no integrada a atención primaria y las carve-in o integradas que genera un cambio sustancial del modelo de atención en salud evitando la fragmentación de esta, considerándose este último más beneficioso para los pacientes. (Wagner, 1998).

Ejemplos exitosos de estos programas son las HMO Lovelace Health Systems y Kaiser Health Plan, quienes consiguieron con la implementación de estos programas ahorros significativos por la reducción de las exacerbaciones y las hospitalizaciones. (Moreno, 2003). En cuanto a Kaiser Permanente, la mayor organización privada para la atención de la salud en Estados Unidos, considera las hospitalizaciones de los pacientes con enfermedades crónicas como una falla en el proceso, pues su modelo ha fomentado grandes centros de atención primaria con capacidad resolutiva, con médicos generales potenciados con perfiles de especialistas, con tecnología para diagnóstico y tratamiento, logrando que las barreras entre los niveles se difumine, con especial atención a la salud poblacional, el compromiso con la difusión del conocimiento de las mejores prácticas, la 
educación continuada y el especial énfasis a la prevención, la detección precoz y la educación sanitaria priorizando la calidad clínica y servicios de urgencia con alta capacidad resolutiva para minimizar hospitalizaciones, los equipos de atención intrahospitalarios integrales planifican el curso de la atención optimizando los recursos institucionales, con cumplimiento riguroso de las programaciones de quirófano y favoreciendo el acceso oportuno al alta hospitalaria ya sea definitiva o a través del cuidado domiciliario, además cuenta con un sistema de información que le da el soporte necesario para favorecer la retroalimentación oportuna (Solinís, 2007).

Lovelace Health Systems- LHS, desarrolló una integración vertical y horizontal, su programa inicia por la búsqueda en la base de datos de las condiciones de salud que explican el mayor costo y utilización, por lo que identificaron 30 patologías para las que desarrollaron programas de gestión de la enfermedad, hicieron análisis de registros médicos tanto ambulatorios como hospitalarios, facturas, dispensación de medicamentos y con un enfoque multifacético que involucra la educación, la reestructura de procesos, fomentando comunicación y uso de herramientas o estrategias comunicativas no sólo con el equipo de salud, sino con el paciente y su familia incluyendo la oportuna retroalimentación de los resultados (Friedman et al., 1999).

A través de la gestión de enfermedades, en los sistemas sanitarios se busca disminuir en el largo plazo los costes por mejora de la eficiencia de la atención, con un buen nivel de calidad por la disminución del consumo de los recursos más costosos, la atención intrahospitalaria. Pasar de atención de condiciones agudas en enfermedades crónicas a la prevención de las mismas que debe mejorar el nivel de salud de una población y por consiguiente a una disminución del uso de servicios hospitalarios a mediano y largo plazo (Terol et al., 2001). El papel protagónico del paciente en estos programas de gestión de la enfermedad, así como de la relación médico- paciente, la importancia del autocuidado y la seguridad que pueden conseguir control de su enfermedad y la implementación de hábitos saludables de vida, hace que el rol de la enfermera en el proceso de atención muestre gran efectividad (Coleman et al., 2009).

La gestión de la enfermedad se concentra en esa porción de la población afectada con determinada patología crónica y entre quienes tienen mayor riesgo de complicaciones, para apalancar los resultados, en la eficiencia en la atención y disminución de la utilización por la mejoría en su estado de salud (Simcoe et al., 2019); pero de acuerdo con Bodenheimer (1999), que mejores resultados en la reducción de morbilidad y mortalidad se obtendrán al involucrar un mayor número de personas con bajo riesgo. Podemos encontrar grandes similitudes en las implementaciones en Colombia de las distintas EAPB, antes llamadas EPS, aun así, los gastos por servicios médicos persisten elevados haciendo parecer insuficiente la Unidad de Pago por Capitación (UPC), que es el valor reconocido por usuario para la cobertura de los planes de beneficio vigentes, aumentando el déficit del sistema y abocando a crisis financiera a muchas IPS las cuales pareciera han apalancado este déficit de cierta forma con las copiosas cartera adeudadas por las EAPB.

\section{Gestión del riesgo en salud}

Desde el 2013 en Colombia, en el Plan Decenal de Salud Pública 2012-2021 (Ministerio de Salud y Protección Social, 2013a) se introduce la gestión de riesgo en salud como una 
estrategia para abordar las necesidades de salud de la población, posteriormente en 2016, con la adopción de la Política de Atención Integral en Salud denominada PAIS, operativizada inicialmente por el Modelo Integral de Atención en Salud- MIAS (Ministerio de Salud y Protección Social, 2016a) y que fue modificado en 2019 al Modelo de Acción Integral Territorial- MAITE (Ministerio De Salud y Protección Social, 2019), se consolida la gestión integral del riesgo en salud como una de las bases sobre la cual pretende la mejora del sistema en términos de satisfacción del usuario, calidad de atención y sostenibilidad del mismo.

En este punto vale la pena profundizar en la definición de riesgo en salud que de acuerdo con el PDSP vigente en Colombia es la probabilidad de ocurrencia de un evento no deseado, evitable y negativo para la salud del individuo, que puede ser también el empeoramiento de una condición previa o la necesidad de requerir más consumo de bienes y servicios que hubiera podido evitarse (Ministerio de Salud y Protección Social, 2013b).

Por lo tanto, la gestión del riesgo en salud será aquella que implemente acciones que disminuyan la materialización de la enfermedad o las complicaciones de esta en la población a cargo. Pretende estar a la delantera de la enfermedad y sus complicaciones, por acciones articuladas entre los diferentes actores del sistema de salud; la identificación y estimación de prioridades relacionadas con grupos de riesgo, a través de intervenciones colectivas, individuales o de autocuidado, de acuerdo con el rol de cada actor (Ministerio de Salud y Protección Social, 2017a).

En la gestión del riesgo en salud se consideran básicamente dos tipos de riesgos, el riesgo primario, que es la posibilidad para cada individuo de aparición de enfermedad y el riesgo técnico relacionado con la calidad de la atención de los servicios de salud, los cuales deben ser identificados, ponderados y abordados por los distintos actores del sistema que tienen a su cargo grupos poblacionales. Implementando y adaptando las RIAS, por el seguimiento y monitoreo de los grupos de riesgo identificados, la aplicación de acciones para manejar los riesgos detectados, se podrá favorecer la integralidad de la atención. Las distintas intervenciones deben ser realizadas por cada uno de los actores desde su rol particular, la colectiva por la entidad territorial, las individuales por la EAPB y las IPS en lo relacionado a la gestión clínica y el autocuidado por parte de cada persona (Ministerio de Salud y Protección Social, 2017a).

Una apropiada gestión del riesgo en salud blindaría a las comunidades frente al deterioro de su desarrollo socioeconómico por favorecer la productividad al disminuir la carga de enfermedad, discapacidad y muerte precoz de los grupos poblacionales de riesgo gestionados.

Esto por cuatro pasos básicos: la identificación de los riesgos, análisis de estos de manera individual y comunitaria, el cálculo del impacto social y económico de la enfermedad y la implementación de acciones que manejen el riesgo identificado. En el contexto colombiano incluyen acciones de: análisis de riesgo de la población con enfoque diferencial, planeación de la atención según riesgos con enfoque diferencial, modificación y vigilancia del riesgo, gestión de la calidad de la atención, coordinación administrativa técnica de los servicios y planes (Rivadeneira, 2014). 
La gestión del riesgo en salud, no debe ser simplificada a un aspecto individual relacionado exclusivamente con la posibilidad de padecer algún evento en salud, ya que se extiende a otros aspectos como el organizacional modulando actuaciones y relaciones entre aseguradores y prestadores, así como el financiero y empresarial de los distintos actores del sistema ya que interactúan para favorecer o entorpecer los resultados en salud de manera individual y colectiva (Jiménez Jaramillo, 2018).

Son dos perspectivas con algo de similitud una vez establecida la enfermedad, podría decirse, que la gestión del riesgo en salud incluye la gestión de la enfermedad, pues por su naturaleza ampliamente anticipatoria y preventiva, en la gestión de la enfermedad están segmentados, es decir, la inclusión dependerá de estar afectado por la patología y en la gestión de riesgo es universal, parte de la caracterización del riesgo individual y colectivo de verse afectado en el curso de la vida por la patología o de estar afectado al momento de la valoración, la intervención en salud se hará previo y durante para anticiparse a las complicaciones (gestión del riesgo) o posterior al diagnóstico (gestión de la enfermedad) e involucrando el total de actores del sistema o un número parcial de acuerdo con el sistema de gestión, la tabla 1 nos amplia este paralelo.

Tabla 1. Paralelo entre gestión de la enfermedad y gestión del riesgo en salud

\begin{tabular}{|c|c|c|}
\hline Detalle & Gestión de la enfermedad & Gestión del riesgo en salud \\
\hline $\begin{array}{l}\text { Grupos } \\
\text { poblacionales } \\
\text { objeto }\end{array}$ & $\begin{array}{l}\text { Segmentado: los afectados por } \\
\text { la enfermedad en mayor o } \\
\text { menor grado. }\end{array}$ & $\begin{array}{l}\text { Universales: incluye los sanos con riesgo de } \\
\text { enfermar y los ya afectados. }\end{array}$ \\
\hline $\begin{array}{l}\text { Momento de la } \\
\text { intervención }\end{array}$ & $\begin{array}{l}\text { Una vez diagnosticada la } \\
\text { enfermedad }\end{array}$ & $\begin{array}{l}\text { Antes de la aparición de la enfermedad y } \\
\text { durante la misma. }\end{array}$ \\
\hline $\begin{array}{l}\text { Análisis de los } \\
\text { grupos } \\
\text { poblacionales }\end{array}$ & $\begin{array}{l}\text { Desde el gasto médico, a través } \\
\text { de históricos de consumo de } \\
\text { atenciones en salud }\end{array}$ & $\begin{array}{l}\text { Desde el mayor riesgo de enfermar por } \\
\text { grupos priorizados adaptados por } \\
\text { regiones, con proyecciones de consumo, } \\
\text { producto de la caracterización individual y } \\
\text { colectiva. Dependiendo la exposición a } \\
\text { factores de riesgo y de protección de la } \\
\text { población. }\end{array}$ \\
\hline $\begin{array}{l}\text { Actores } \\
\text { involucrados }\end{array}$ & $\begin{array}{l}\text { Asegurador y prestador activos } \\
\text { y en un papel pasivo el usuario }\end{array}$ & $\begin{array}{l}\text { Todos los actores del sistema con un rol } \\
\text { activo: entidades territoriales, } \\
\text { aseguradores, prestadores y el usuario }\end{array}$ \\
\hline $\begin{array}{l}\text { Enfoque } \\
\text { Tipo de } \\
\text { intervenciones }\end{array}$ & $\begin{array}{l}\text { Reactivo y de contención } \\
\text { Individuales y de fomento del } \\
\text { autocuidado }\end{array}$ & $\begin{array}{l}\text { Anticipatorio y preventivo. } \\
\text { Colectivas, individuales y de fomento del } \\
\text { autocuidado, articuladas entre los distintos } \\
\text { actores }\end{array}$ \\
\hline
\end{tabular}

Fuente: Elaboración propia

\section{CLAVES PARA EL DISEÑO E IMPLEMENTACIÓN DE UN SISTEMA DE GESTIÓN DE RIESGO EN UNA INSTITUCIÓN PRESTADORA DE SERVICIOS DE SALUD EN COLOMBIA}

Hay un gran campo por explorar en lo relacionado con la gestión del riesgo en salud en Colombia y en especial en las IPS, las metodologías más utilizadas coinciden en lo 
trascendente del análisis de la situación en todos los aspectos, internos y externos, así como en el despliegue de la información a todos los niveles de la organización. La mayoría de las IPS en Colombia han implementado la política y el programa de seguridad del paciente, lo que cubre en su gran parte los riesgos asociados a la atención en salud. La propuesta planteada para el diseño e implementación de la gestión del riesgo, se basa en involucrar aquellos riesgos extrapolados de los sectores bancario y el asegurador, algunos de los cuales son contemplados por las guías para la administración del riesgo expedidas por el Departamento Administrativo de la Función Pública como son: el riesgo reputacional, los riesgos de gobierno corporativo, los riesgos de mercado, el riesgo del modelo de negocio, el riesgo de crédito, el operacional, los riesgos comerciales y por supuesto el riesgo financiero, el riesgo informático y el de corrupción. Siendo que aún no han sido definidos por la Superintendencia Nacional de Salud, los riesgos específicos para las IPS deberán ser incluidos en la normativa, para generarse el control pertinente por parte de ese ente., de forma independiente a que se expida dicha normativa.

La gestión del riesgo debe ser liderada y función específica de los directores o gerentes de las IPS para favorecer el crecimiento, para conquistar los objetivos planteados controlando sus procesos con medidas efectivas, logrando detectar y controlar todo aquello que pueda afectarlos. Las IPS deben aumentar su rendimiento, ser más eficaces y eficientes, mejorando el control al interior, adjudicando convenientemente los recursos, el manejo de la información, incrementando la confianza para la toma de decisiones y su planeación. Así mismo, se debe el pleno cumplimiento legal y no menos importante el riesgo reputacional.; se necesitan equipos directivos bien estructurados, con conocimiento y experiencia, que lideren la gestión de los riesgos en las IPS de manera organizada y metódica, de tal forma que aborden la mayoría de los riesgos aquí planteados.

A futuro se sugiere realizar trabajos pilotos de caracterización en IPS reconocidas como casos exitosos al implementar y sostener un robusto sistema de gestión del riesgo teniendo en cuenta la gestión del riesgo en salud sin restarle importancia a la gestión de la enfermedad. Suscitar en los líderes de las IPS, profundo interés por investigar y proponer nuevas estrategias para la gestión de los riesgos y por tanto nuevas metodologías.

\section{REFERENCIAS}

Arbeláez, J. C., Franco, L. C., Betancur, C., Murillo, J. G., Gallego, P. A., Henao, V. M., Londoño, J. A., Mejía, C. M., Palacio, D. M., Salazar, E., Salazar, L. F., Valderrama, N., \& Varela, D. C. (2006). Riesgo operacional: Reto actual de las entidades financieras. Revista Ingenierías Universidad de Medellín, 5(9), 97-110.

Assi, M. (2012). Gestão de riscos com controles internos: Ferramentas, certificações e métodos para garantir a eficiência dos negocios. Saint Paul Editora.

Barros, R. H. \& de Madrid. (2011). Metodología financiera de gestión y cuantificación de riesgos de las entidades aseguradoras. 27.

Becerra, L. A. C., \& Semper, J. D. C. (2007). Las opciones financieras como mecanismo para estimar las primas de seguro y reaseguro en el sistema de salud colombiano. 16. 
Bodenheimer, T. (1999). Disease Management_Promises and Pitfalls. The New England Journal of Medicine, 340(15), 1202-1205.

Care Continuum Alliance (CCA). (s. f.). Care Continuum Alliance | Disease Management Defined. Recuperado 10 de junio de 2020, de http://www.carecontinuum.org/dm_definition.asp

Coleman, K., Mattke, S., Perrault, P. J., \& Wagner, E. H. (2009). Untangling practice redesign from disease management: How do we best care for the chronically ill? Annual review of public health, $30,385-408$.

Congreso de la República de Colombia. (1993, Diciembre 23). Ley 100 de 1993. Por la cual se crea el sistema de seguridad social integral y se dictan otras disposiciones. DO: 41.148.

Congreso de la República de Colombia. (2007, Enero 9). Ley 1122 de 2007. Por la cual se hacen algunas modificaciones en el sistema general de seguridad social en salud y se dictan otras disposiciones. DO: 46.506.

Congreso de la República de Colombia. (2011, Enero 19). Ley 1438 de 2011. Por medio de la cual se reforma el sistema general de seguridad social en salud y se dictan otras disposiciones. DO: 47.957.

Congreso de la República de Colombia. (2015, Febrero 16). Ley estatutaria 1751 de 2015. Por medio de la cual se regula el derecho fundamental a la salud y se dictan otras disposiciones. DO: 49.427.

Congreso de la República de Colombia. (2019, Mayo 25). Ley 1955 de 2019. Por el cual se expide el Plan Nacional de Desarrollo 2018 -2022" Pacto por Colombia, Pacto por la Equidad. DO: 50.964.

Constitución Política de Colombia. (1991, Julio 20). GC: 116.

Crouhy, M., Galai, D., \& Mark, R. (2008). Model Risk. En Encyclopedia of Quantitative Risk Analysis and Assessment. American Cáncer Society. https://doi.org/10.1002/9780470061596.risk0385

De Oliveira, L. M., Silva De Oliveira, A. M., De Oliveira, A., \& Lopes Da Gama, P. S. (2008). Contabilidade internacional: Gestão de riscos, governança corporativa e contabilização de derivativos. Atlas.

Departamento Administrativo de la Función Pública. (2014). Guía para la administración del riesgo.

Departamento Administrativo de la Función Pública. (2018). Guía para la administración del riesgo y el diseño de controles en entidades públicas. Riesgos de gestión, corrupción y seguridad digital.

Friedman, N., Skogmo, L., \& Aruffo, P. (1999). Implementing disease management programmes within an integrated delivery system. Disease Management and Health Outcomes, 6(6), 355-363. Scopus. https://doi.org/10.2165/00115677-199906060-00007

Gaviria, L. M. P., \& Lemarie, A. L. (2008). Administración del riesgo operacional en Colombia. 19.

Gil, A. de L., Arima, C. H., \& Nakamura, W. T. (2013). Gestão: Controle interno, risco e auditoria. Saraiva.

Giraldo, A. L. P., \& Núñez, M. A. (2020). Administración Del Riesgo Estratégico En Algunas Grandes Empresas Privadas De Colombia Estratégic Risk Management In Some Large Colombian Private Companies. 31.

Hammer, M., Champy, J., \& Korytowski, I. (1994). Reengenharia: Evolucionando a empresa em função dos clientes, da concorrencia e das grandes mudanças da gerencia (29.a ed.). Campus.

Instituto Brasileiro De Governança Corporativa. (2007). Guía de Orientação para Gerenciamento de Riscos Corporativos. www.ibgc.org.br 
Instituto Colombiano de Normas Técnicas y Certificación. ICONTEC. (2011). NTC- ISO31000:2011 Gestión del riesgo. Principios y directrices (Instituto Colombiano de Normas Técnicas y Certificación. ICONTEC).

ISOTools. (2018, Noviembre 28). Norma ISO 31000: El valor de la gestión de riesgos en las organizaciones. www.isotools.org: https://www.isotools.org/pdfs-pro/ebook-iso-31000-gestionriesgos-organizaciones.pdf

Jiménez Jaramillo, C. A. (2018). Qué importancia tiene la gestión integral del riesgo en salud en la viabilidad financiera del Sistema General de Seguridad Social? [Master's Thesis]. Universidad EAFIT.

Jiménez Rodríguez, E. J., \& Martín Marín, J. L. (2005). El nuevo acuerdo de Basilea y la gestión del riesgo operacional. UCJC Business and Society Review, 3(7), 54-67.

Jorion, P. (1998). Value at Risk: A nova fonte de referência para o controle do risco de mercado. . Bolsa de Mercadorias \& Futuros.

Marshall, C. L. (2002). Medindo e Gerenciando Riscos Operacionais em Instituições Financeiras. Qualitymark Editora.

Ministerio de Salud y Protección Social. (2013a, junio 4). Resolución 1841 de 2013. Por la cual se adopta el Plan Decenal de Salud Pública 2012-2021. DO: 48.811.

Ministerio de Salud y Protección Social. (2013b). ABC del Plan Decenal de Salud Pública. Imprenta Nacional de Colombia. https://www.minsalud.gov.co/sites/rid/Lists/BibliotecaDigital/RIDE/VS/ED/PSP/IMP_4feb+ABCm insalud.pdf

Ministerio de Salud y Protección Social. (2014, Mayo 30). Resolución 2082 de 2014. Por la cual se dictan disposiciones para la operatividad del sistema único de acreditación en salud. DO: 49.167.

Ministerio de Salud y Protección Social. (2015a, de Febrero de). Resolución 518 de 2015. Por la cual se dictan disposiciones en relación con la Gestión de la Salud Pública y se establecen directrices para la ejecución, seguimiento y evaluación del Plan de Salud Pública de Intervenciones Colectivas (PIC). DO: 49.436.

Ministerio de Salud y Protección Social. (2015b, mayo 11). Resolución 1536 de 2015. Por la cual se establecen disposiciones sobre el proceso de planeación integral para la salud. DO: 49.508.

Ministerio de Salud y Protección Social. (2016a, febrero 22). Resolución 429 de 2016. Por medio de la cual se adopta la Política de Atención Integral en Salud. DO: 49.794.

Ministerio de Salud y Protección Social. (2016b, julio 27). Resolución 3202 de 2016. Por la cual se adopta el Manual Metodológico para la elaboración e implementación de las Rutas Integrales de Atención en Salud (RIAS), se adopta un grupo de Rutas Integrales de Atención en Salud desarrolladas por el Ministerio de Salud y Protección Social. DO: 49.947.

Ministerio de Salud y Protección Social. (2017a). Capítulo 3 Gestión Integral del Riesgo. En Modelo Integral de Atención en Salud Cartilla guía. Ministerio de Salud y Protección Social.

Ministerio de Salud y Protección Social. (2017b). Cartilla Gestión Integral del Riesgo.pdf. En Modelo Integral de Atención en Salud Cartilla guía.

Ministerio de Salud y Protección Social. (2018, Junio 18). Resolución 2515 de 2018. Por medio de la cual se reglamentan las condiciones de habilitación de las entidades responsables de la operación del aseguramiento en salud y los estándares de oportunidad y acceso para la operación territorial del aseguramiento. DO: 50.628. 
Ministerio De Salud y Protección Social. (2019, Septiembre 30). Resolución 2626 de 2019. Por la cual se modifica la Política de Atención Integral en Salud (PAIS) y se adopta el Modelo de Acción Integral Territorial (Maite). DO: 51.092.

Ministerio de Salud y Protección Social. (2019, Noviembre 26). Resolución 3100 de 2019. Por la cual se definen los procedimientos y condiciones de inscripción de los prestadores de servicios de salud y de habilitación de los servicios de salud y se adopta el Manual de Inscripción de Prestadores y Habilitación de Servicios de Salud. DO: 51.149.

Ministerio de Salud y Protección Social. (2020, Julio 8). Resolución 1147 de 2020. Por la cual se modifican los artículos 9 y 10 de la Resolución 2626 de 2019 en relación con la aplicación de unos plazos. D.O: 51370 .

Moreno, S. P. (2003). De la gestión de lo complementario a la gestión integral de la atención de salud: Gestión de enfermedades e indicadores de actividad. En Gestión clínica y sanitaria: De la práctica a la academia, ida y vuelta (pp. 17-87). Masson.

Nunes, R. P. (2009). Analise do Fluxo de Caixa em Risco para uma Empresa Productora de Derivados de Petróleo [PhD Thesis]. PUC-Rio.

OMS/BIREME/OPS. (2019, Mayo 3). Descriptores en ciencias de la salud DeCS/MeSH 2018—Versión 31 de julio de 2018. Descriptores en ciencias de la salud DeCS 2018-Versión 31 de julio de 2018. https://decs.bvsalud.org/es/

Pérez-Fructuoso, M. J. (2014). Implicaciones en la gestión del riesgo de los acuerdos de Solvencia II y Basilea III.

Presidencia de la República de Colombia. (2015, Mayo 26). Decreto 1083 de 2015. Decreto Único Reglamentario del Sector de Función Pública. DO: 49.523.

Presidencia de la República de Colombia. (2016, Mayo 6). Decreto 780 de 2016. Decreto Único Reglamentario del Sector Salud y Protección Social. DO: 49.865.

Rivadeneira, A. G. (2014). Marco conceptual y legal sobre la gestión de riesgo en Colombia: Aportes para su implementación. Monitor Estratégico, 5, 4-11.

Rodrigues Pereira, M. (2014, marzo). O Gerenciamento De Riscos Empresariais Como Forma De Agregar Valor Às Organizações. https://pantheon.ufrj.br/bitstream/11422/1666/1/MRPereira.pdf. https://pantheon.ufrj.br/bitstream/11422/1666/1/MRPereira.pdf

Sánchez, L. R. (2016). COSO ERM y la gestión de riesgos. Quipukamayoc, 23(44), Article 44. https://doi.org/10.15381/quipu.v23i44.11625

Simcoe, T., Catillon, M., \& Gertler, P. (2019). Who benefits most in disease management programs: Improving target efficiency. Health economics, 28(2), 189-203.

Smith, Preston, G., Merritt, \& Guy, M. (2002, septiembre). Managing consulting project risk. Consulting to Management, 13(3), 7-13.

Solinís, R. N. (2007). Buenas prácticas en gestión sanitaria: El caso Káiser Permanente. Revista de Administración Sanitaria, 5(2), 283-292.

Superintendencia Nacional de Salud. (2018a, abril 12). Resolución 4559 de 2018. Por medio de la cual se adopta el modelo de Inspección, Vigilancia y Control para la Superintendencia Nacional de Salud para el ejercicio de la supervisión de los riesgos inherentes al Sistema General de Seguridad Social en Salud. D. O: 50.562.

Superintendencia Nacional de Salud. (2018b, julio 3). Circular Externa 000004 de 2018. Por la cual se imparten instrucciones generales relativas al código de conducta y de buen gobierno 
organizacional, el sistema integrado de gestión de riesgos y a sus subsistemas de administración de riesgos. DO: 50.643 .

Superintendencia Nacional de Salud. (2018c, agosto 3). Circular Externa 000006 de 2018. Por la cual se hacen modificaciones a la circular 047 de 2007 en lo relacionado con el sistema integrado de gestión de riesgos y a sus subsistemas de administración de riesgos, y reporte de información. DO: 50.674 .

Terol, E., Hamby, E. F., \& Minue, S. (2001). Gestión de Enfermedades (Disease Management): Una aproximación integral a la provisión de cuidados sanitarios. Medifam, 11(2), 7-20.

Vega de la Cruz, L. O., de la Caridad Pérez Pravia, M., \& Moreno Pino, M. del R. (2017). El chinchorro de gestión de riesgos como filosofía moderna de mejora en la dirección / The management's fish net of risk as a modern philosophy of improvement in administration. Pensamiento \& Gestión, 43, 66-66-88. SciELO. https://doi.org/10.14482/pege.43.10581

Wagner, E. H. (1998). Chronic disease management: What will it take to improve care for chronic illness? Effective clinical practice, 1(1), 2-4.

Zeno, J. M. da C. (2007). Risco legal: Uma introdução ao seu gerenciamento no atual cenário corporativo. [Dissertação de Mestrado Profissionalizante em Administração.]. Faculdades Ibmec. 